\title{
Cholecystokinin-B/gastrin receptors enhance wound healing in the rat gastric mucosa
}

\author{
Adrian Schmassmann ${ }^{1}$ and Jean Claude Reubi ${ }^{2}$ \\ ${ }^{1}$ Gastrointestinal Unit, Inselspital, University Hospital, and \\ ${ }^{2}$ Division of Cell Biology and Experimental Cancer Research, Institute of Pathology, University of Berne, Berne, Switzerland \\ Address correspondence to: A. Schmassmann, Gastrointestinal Unit, Inselspital, University Hospital, 3010 Berne, Switzerland. \\ Phone: 001-41-31-632 8025; Fax: 001-41-31-632 9765; E-mail: adrian.schmassmann@kss.gsd.lu.ch. \\ Received for publication May 19, 2000, and accepted in revised form September 5, 2000.
}

\begin{abstract}
Although physiological functions of the CCK-B/gastrin receptor are well explored, little is known about its role during healing. Here, we evaluated the role of this receptor in the rat oxyntic mucosa following the introduction of a cryoulcer. In this model, we located and quantified CCK-B/gastrin receptors by reverse transcriptase PCR and receptor autoradiography. Rats with cryoulcers were treated with placebo, omeprazole, the CCK-B/gastrin receptor antagonist YF-476, omeprazole plus YF476, gastrin-17, and gastrin 17 plus YF-476. During wound healing, CCK-B/gastrin receptors were specifically expressed and localized to the regenerative mucosal ulcer margin. This high expression was limited in time, and the pattern of expression of CCK-B/gastrin receptors correlated closely with the proliferative activity of the regenerative mucosa. Functionally, omeprazole and gastrin-17 caused profound hypergastrinemia, increased cell proliferation in the mucosal ulcer margin and accelerated the late ulcer healing phase. These effects were completely reversed by cotherapy with YF-476. These in vivo and vitro data suggest that CCK-B/gastrin receptors in regenerative rat gastric oxyntic mucosa enhance trophic effects during wound healing.
\end{abstract}

J. Clin. Invest. 106:1021-1029 (2000).

\section{Introduction}

The wound-healing process, both in humans and in experimental ulcer models, is a complex process requiring the collaborative efforts of many different tissues and cell lineages. The behavior of each of the contributing cell types during the phases of proliferation, migration, matrix synthesis, and contraction, as well as the presence of growth factor and matrix signals present at a wound site, are now roughly understood (1-6). In contrast to the role of growth factor receptors, the role of gut hormone receptors during healing is less clear. Therefore, the characterization of hormone receptors that can be therapeutically influenced is important $(7,8)$. Stimulation of wound healing by small molecules that directly or indirectly influence hormone receptors is an attractive strategy to improve wound healing in several organ systems (1, 7-9).

The cholecystokinin-B/gastrin receptor (CCK-BR) mediates the actions of gastrin and CCK in the gut and the brain. It can be inhibited by potent CCK-BR antagonists such as YM022 (10), YF-476 (11), and S0509 (12), or indirectly stimulated by acid-inhibitory drugs such as omeprazole, which causes endogenous hypergastrinemia through the increased release of various forms of gastrin from antral G cells $(3,13,14)$. Whereas the physiological role of gastrin and the CCK$\mathrm{BR}$ has been rather well characterized (15-18), little is known about a possible role of gastrin and the CCK$\mathrm{BR}$ in healing. In vivo studies using gastric woundhealing models have shown that cell proliferation in regenerative mucosa is increased in hypergastrinemic (19) and decreased in gastrin-depleted states (20). These studies have supported the concept that gastrin, whether triggered by endogenous mechanisms (i.e., omeprazole treatment) or exogenous hypergastrinemia (i.e., infusion of gastrin-17), stimulates cell proliferation in regenerative gastric mucosa (19). However, the molecular mechanisms that mediate these actions have not yet been identified or characterized, either in vitro or in vivo. Thus, it is not known whether the action of gastrin is a direct one at the repair site, possibly mediated through CCK receptors, the low-affinity gastrin-binding protein (21), or other distinct and not yet clearly defined receptors (22-24), or whether it acts indirectly, for instance through the stimulation of other growth factors (25).

To study the role of CCK receptors during wound healing, we selected a well-defined and established cryoulcer model in rats in which the in vivo effect of gastrin on regenerative mucosa can be quantified $(3,4,19$, 20). This model has been extensively validated by evaluating a substantial number of various parameters, such as the expression of growth factors and their receptors, enzymes, and secretory products $(2-6,19$, 20, 26-28). Furthermore, the influence of gastric acidity on ulcer-healing mechanisms has been extensively studied in this model $(3,4)$. This is important, since omeprazole and YF-476 nearly abolish gastric acid secretion, whereas gastrin-17 persistently increases gastric acid secretion. 
The aims of this study were to (a) test whether CCK receptors are expressed in gastric repair tissue by using RT-PCR and receptor autoradiography, (b) assess the location and time sequence of the expression of these CCK receptors, (c) characterize the CCK receptor subtype, (d) quantify the in vivo trophic effects of gastrin on ulcer healing and epithelial cell proliferation in the mucosal ulcer margin, and (e) test whether YF-476 can reverse these gastrin effects. These data should answer the basic question of whether the CCK-BRs enhance trophic effects during wound healing.

\section{Methods}

The study was approved by the Animal Study Committee of the University of Berne. Standardized gastric ulcers were produced in the oxyntic mucosa of female Wistar rats by a cryoprobe as described previously $(3,4)$.

$R T$-PCR. Rats with cryoulcers were sacrificed $1,3,8$, and 15 days (12 rats per time point) after ulcer induction. Regenerative mucosa was carefully removed and immediately frozen in liquid nitrogen. The sampling technique was optimized to minimize contamination of the regenerative mucosa with normal mucosa and controlled by histological examinations of selected samples (28). The total RNA was isolated from tissues by the RNeasy kit of QIAGEN (Basel, Switzerland). Contaminating DNA was then removed by incubating with DNaseI (Boehringer, Mannheim, Germany) and applying the RNeasy procedure. An aliquot of the RNA was used to determine the concentration by measuring the OD at $260 \mathrm{~nm}$. The results of an agarose gel electrophoresis showed that the isolated RNA was intact. Total $(4 \mu \mathrm{g})$ RNA was reverse transcribed using $20 \mathrm{U}$ of murine leukemia virus reverse transcriptase (Boehringer) in $40 \mu \mathrm{l}$ reaction volume (buffer provided by manufacturer, $1 \mathrm{mM}$ dNTP, $0.08 \mu \mathrm{M}$ primer $\mathrm{dT}_{15}$, $20 \mathrm{U}$ RNase inhibitor). After 2 hours at $37^{\circ} \mathrm{C}$, the reaction was stopped by incubating at $70^{\circ} \mathrm{C}$ for 10 minutes, then was diluted to $400 \mu \mathrm{l}$ and frozen in aliquots. As a negative control, RNA from normal mucosa was used in an identical procedure, but without adding the reverse transcriptase. For PCR reactions, primers were designed using sequences taken from the Genbank. Reactions were performed using Advantage Polymerase mix (CLONTECH Laboratories, Palo Alto, California, USA). Cycling conditions were 30 seconds at $94^{\circ} \mathrm{C}, 30$ seconds at annealing temperature, and 60 seconds at $72^{\circ} \mathrm{C}$. In pilot experiments with increasing cycle numbers, the amplification cycle was determined, which produced DNA bands in a phase of nearly linear DNA production below the maximal possible yield. The PCR products were analyzed on a $1 \%$ agarose gel containing ethidium bromide.

Primers, annealing temperatures, cycle numbers, and product sizes used in the PCR reactions were as follows: (a) for CCK-BR, forward primer: 5'-GAGCCGACGCCTAAGAACGGTC-3'; backward primer: 5'-GTGCATGCATCGCTGGCCCAGT-3'; annealing temperature: $55^{\circ} \mathrm{C}$; cycle number: 30; product size: $391 \mathrm{bp}$; (b) for met, for- ward primer: 5'-CTATATGAAGCACGGAGATCTTCG-3'; backward primer: $5^{\prime}$-AGAGAAGCACACCGAAGGACCAC3'; annealing temperature: $57^{\circ} \mathrm{C}$; cycle number: 30 ; product size: $347 \mathrm{bp}$; (c) pepsinogen, forward primer: 5'-CAGACCTTCTCCCTGCAGTACG-3'; backward primer: 5'-AAACACAATCTGCCCACCGTTAG-3'; annealing temperature: $55^{\circ} \mathrm{C}$; cycle number: 18 ; product size: $300 \mathrm{bp}$; (d) for cyclooxygenase-2 (COX-2), forward primer: 5'TTACCACTTCAAACTCAAGTTCG-3'; backward primer: 5'CGTTTGCGGTACTCATTGAGAGAC-3'; annealing temperature: $55^{\circ} \mathrm{C}$; cycle number: 34 ; product size: $345 \mathrm{bp}$; and (e) for GAPDH, used as a reference gene, forward primer: 5'-GTGTGAACGGATTTGGCCGTATC-3'; backward primer: 5'-TTCTTGATGTCATCATACTTGGC-3'; annealing temperature: $55^{\circ} \mathrm{C}$; cycle number: 24 ; product size: $760 \mathrm{bp}$. The intensities of the bands were quantified by densitometry using a computer-coupled video camera and the Image-Pro software (Media Cybernetics, Silver Spring, Maryland, USA). Values for each gel were normalized by dividing all values by the mean of the intensities of the values from day 1 . The correct identity of the amplification products was confirmed by sequencing them.

In situ bybridization and immunobistochemistry. Control rats and rats with cryoulcers (three rats per time point) were sacrifice $1,3,8,15$, and 84 days after ulcer induction. Gastric specimens were fixed for 4 hours in $4 \%$ buffered paraformaldehyde, dehydrated gradually in ethanol, embedded in paraffin, and then in situ hybridization was performed as described previously $(6,29,30)$. In brief, a cDNA fragment corresponding to nucleotides 514-816 of the rat pepsinogen type C cDNA (Genbank accession no. X04644) was produced by reverse transcription and PCR using rat stomach RNA (29). Linearized DNA templates were generated by PCR using 5 ng of plasmid DNA and primers covering the SP6-and T7-recognition region of the plasmid. About $2 \mu \mathrm{g}$ of linear DNA were thus obtained, and it was further purified using a QIAGEN spin column. Digoxigenin-labeled antisense and sense RNA probes were then made by in vitro transcription with either $\mathrm{T} 7$ or SP6 RNA polymerase using chemicals and manuals provided by Boehringer. The integrity and amount of the riboprobes were assessed by using agarose gel electrophoresis. In situ hybridizations were performed at $55^{\circ} \mathrm{C}$ with $35 \mathrm{ng} / \mathrm{ml} \mathrm{RNA}$ probes (29). Detection of the hybrid RNA was with Ab's against digoxigenin coupled to alkaline phosphatase using nitroblue tetrazolium and 5-bromo-4-chloro-3-indolyl-phosphate as substrates. Sections were then stained further with the mAb 2G11 against $\mathrm{H}^{+}, \mathrm{K}^{+}$-ATPase as previously described (3). Bound Ab's were made visible by using biotin-coupled goat anti-mouse-IgA plus IgG plus IgM Ab's, streptavidin-coupled horseradish peroxidase, and the chromogen 3-amino-9-ethyl-carbazole. In situ hybridization for met was performed as described (4).

Receptor autoradiography. Rats with cryoulcers were sacrificed $3,5,8,15,22,29,56$, and 84 days after ulcer induction (three to seven rats per time point). The 
stomach wall containing the ulcerated tissue or the mucosal scar was frozen on dry ice and stored at $-70^{\circ} \mathrm{C}$, as previously described $(17,31,32)$. Receptor autoradiography was performed on 10 - and $20-\mu \mathrm{m}$-thick cryostat sections (Leitz 1720; Ernst Leitz Wetzlar Gmbh, Wetzlar, Germany) of the tissue samples mounted on microscope slides, and then stored at $-20^{\circ} \mathrm{C}$ for at least 3 days, as described previously, using ${ }^{125} \mathrm{I}-\mathrm{D}-\mathrm{Tyr}-\mathrm{Gly}-$ Asp-Tyr(SO3H)-Nle-Gly-Trp-Nle-Asp-Phe-amide ( ${ }^{125} \mathrm{I}$ CCK) as a radioligand $(17,31)$. The sections were preincubated in $50 \mathrm{mmol} / 1$ Tris- $\mathrm{HCl}, 130 \mathrm{mmol} / \mathrm{l} \mathrm{NaCl}, 4.7$ $\mathrm{mmol} / \mathrm{l} \mathrm{KCl}, 5 \mathrm{mmol} / \mathrm{l} \mathrm{MgCl}, 1 \mathrm{mmol} / \mathrm{l}$ EGTA, and $0.5 \% \mathrm{BSA}, \mathrm{pH} 7.4$ (preincubation solution), for $30 \mathrm{~min}-$ utes at $25^{\circ} \mathrm{C}$. The slides were then incubated in a solution containing the same medium as the preincubation solution, except that the BSA was omitted and the following compounds were added: $0.025 \%$ bacitracin, 1 $\mathrm{mmol} / \mathrm{l}$ dithiothreitol, $2 \mu \mathrm{g} / \mathrm{ml}$ chymostatin, $4 \mu \mathrm{g} / \mathrm{ml}$ leupeptin, $\mathrm{pH}$ 6.5, and the radioligand, $45 \mathrm{pM}^{125} \mathrm{I}-\mathrm{CCK}$ (2000 Ci/mmol; Anawa Trading SA, Wangen, Switzerland). The slides were incubated at room temperature with the radioligand for 150 minutes. Increasing amounts of nonradioactive sulfated CCK-8, gastrin-17, YF-476, and the CCK-A receptor (CCK-AR) antagonist L-364,718 were added to the incubation medium to generate competitive inhibition curves. On completion of the incubation, the slides were washed six times for 15 minutes each in ice-cold preincubation solution, $\mathrm{pH}$ 7.4. They were rinsed twice in ice-cold distilled water for 5 seconds each, and then dried under a stream of cold air at $4^{\circ} \mathrm{C}$, apposed to ${ }^{3} \mathrm{H}$-Hyperfilm, and exposed for 1 to 7 days in $\mathrm{x}$-ray cassettes. The autoradiograms were quantified using a computer-assisted image-processing system (31). Tissue standards for iodinated compounds (Amersham Pharmacia Biotech Europe, Freiburg, Germany) were used for this purpose. The ${ }^{125}$ I-CCK decapeptide identifies the CCK-BR when the ligand is displaced by nanomolar concentrations of CCK, gastrin-17, or YF-476, or it identifies the CCK-AR when the ligand is displaced by nanomolar concentrations of CCK or L-364,718, but not gastrin-17 or YF-

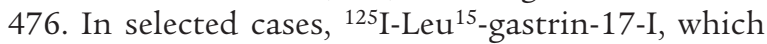
identifies the CCK-BR but not the CCK-AR, was used as radioligand, as described previously (17).

In vivo studies. A gastric cannula, which allowed videoendoscopic examination of the gastric mucosa, was placed into the rumen of female Wistar rats (body weight, 180-200 g) (3, 4). Three weeks later, standardized gastric ulcers were produced by using a cryoprobe, as described previously. Gastric wound healing (ulcerdiameter time curve and ulcer-healing rate time curve) was assessed by repeated video-endoscopic and histologic measurements of ulcer size $(3,4)$. Before the experiments, all animals were deprived of food for 18 hours, and the animals were kept in mesh-bottom cages to prevent coprophagy. Two hundred twentyeight rats with cryoulcers were randomly assigned and treated twice daily subcutaneously with (group 1) placebo, (group 2) omeprazole $(20 \mu \mathrm{mol} / \mathrm{kg})$, (group 3$)$
YF-476 (50 $\mu \mathrm{mol} / \mathrm{kg})$, (group 4) omeprazole (20 $\mu \mathrm{mol} / \mathrm{kg}$ ) plus YF-476 (50 $\mathrm{mol} / \mathrm{kg})$, (group 5) gastrin$17(100 \mathrm{nmol} / \mathrm{kg})$, and (group 6) gastrin 17 (100 $\mathrm{nmol} / \mathrm{kg})$ plus YF-476 $(50 \mu \mathrm{mol} / \mathrm{kg})$ for 3,8 , and 15 days (vol. $0.25 \mathrm{ml}$, given in various sites of the body). The number of rats per treatment group were as follows: 72 rats in group 1, 36 rats each in group 2-4, and 24 rats each in group 5 and 6 . The rats were sacrificed 1 hour after dosing on days 3, 8, and 15. YF-476 (R)-1[2,3-dihydro-2-oxo-1-pivaloylmethyl-5-(2-pyridyl)-1H1,4-benzo-diazepin-3-yl]-3-(3-methylaminophenyl)urea, was kindly provided by Yamanouchi (Osaka, Japan). Omeprazole was obtained from Astra Hässle (Mölndal, Sweden). Placebo consisted of omeprazole solvent. Rat gastrin-17-I was obtained from Bachem AG (Bubendorf, Switzerland) and given as a suspension in olive oil (depot form), as described previously (33). The rats were anesthetized with ether, laparotomized, and the stomach was removed as described $(3,4)$. The ulcerated region on the posterior wall and the intact tissue on the anterior wall were excised, fixed for 4 hours in $4 \%$ buffered paraformaldehyde, dehydrated gradually in ethanol, and embedded in paraffin as previously described $(3,4)$. Compared with placebo, omeprazole and YF-476 persistently inhibited basal and gastrin-stimulated acid secretion, and gastrin-17 substantially increased gastric acid secretion (data not shown). Injection of gastrin-17 in olive oil caused a persistent increase of plasma gastrin levels greater than $300 \mathrm{nmol} / 1$ during the entire treatment period of 15 days.

Cell proliferation was quantified on days 3,8 , and 15 , as described previously (3). In brief, a single intraperitoneal injection of $100 \mathrm{mg} / \mathrm{kg}$ bromodeoxyuridine (BrdU) was administered 1 hour before the stomach was removed. Tissue sections were stained with $\mathrm{mAb}$ against BrdU and bound Ab's were visualized using anti-mouse biotin Ab's, streptavidin-coupled horseradish peroxidase, and fast red as a chromogen (3). The percentage of BrdU-labeled cells was determined in the normal mucosa of the anterior wall and in a $600-\mu \mathrm{m}$-wide region of the mucosa adjacent to the ulcer crater. The percentage of labeled cell nuclei over the total number of counted cell nuclei was calculated. The mean value per rat was determined by examining 20 sections of the normal mucosa and 20 sections of the ulcerated region.

For the quantitative assessment of histological parameters, serial sections ( $5 \mu \mathrm{m}$ thick) containing the total ulcer region on the posterior wall and the intact gastric wall on the anterior wall were cut perpendicular to the surface of the mucosa, and three consecutive sections at a distance of $50 \mu \mathrm{m}$ were taken (3). The slides were quantitatively analyzed with a video camera (Ci-20 P; Canon, Tokyo, Japan) and a semiautomatic image analysis system (Microvid software; Leica, Zürich, Switzerland).

The mucosal ulcer margin was defined as the area containing regenerative mucosa without signs of maturity $(3,4)$. Three consecutive slides per rat were selected that showed the maximal ulcer size and that were there- 
fore most closely located to the center of the ulcer; in these slides, length and average height of the mucosal ulcer margin were measured on both sides of the ulcer. The mean value per rat was determined from six measurements. The length of the mucosal ulcer margin was defined as distance between the ulcer crater and the border of the ulcer margin and the intact oxyntic mucosa. The average height of the ulcer margin was defined as area/length, and the total area of the regenerative mucosa at the ulcer margin was traced as described previously $(3,4)$.

Blood samples were collected before first dosing on day 0 and 1 hour after last dosing. Plasma gastrin levels were measured by RIA as described previously (3). In rats receiving gastrin-17 or gastrin-17 plus YF-476, gastrin plasma levels were averaged from three blood samples within a 12-hour dosing interval on the day before autopsy (taken 2, 6, and 10 hours after dosing).

Differences between groups were tested by ANOVA followed by Student's $t$ test. Correlation analyses were performed according to Pearson. Probability values of $P$ less than 0.05 were regarded as significant. Values are means plus or minus SEM from 6-12 rats in the in vitro studies and $8-24$ rats in the in vivo studies.

\section{Results}

Description and validation of the cryoulcer model in rats. Gastric ulceration was caused by standardized cryoinjury. During the ulcer-repair period, both histological and biochemical parameters were investigated. The proliferative zone in the repair tissue was expanded to comprise the entire length of the glands. Cells in DNA-synthetic phase that were labeled with Ab's against BrdU were substantially increased in the lower part of the glands between days 3 and 15 (Figure 1). Thereafter, the ulcer became completely re-epithelialized and cell proliferation, as a marker of repair activity, decreased rapidly (Table 1). In parallel, marked and rapid dedifferentiation processes occurred after cryoinjury in the repair zone of the mucosal ulcer margin. Parietal and chief cells decreased in size, dedifferentiated, or were replaced by proliferating regenerative cells. In the regenerative mucosa of the repair zone, pepsinogen mRNA (a marker for mature chief cells) and $\mathrm{H}^{+}, \mathrm{K}^{+}$-ATPase protein (a marker for parietal cells) decreased below the detection level of in situ hybridization and immunohistochemistry (Figure 1).

In vitro CCK-BR expression using receptor autoradiography. Specific binding for ${ }^{125} \mathrm{I}-\mathrm{CCK}$ and ${ }^{125} \mathrm{I}$-gastrin was detected in the regenerative mucosa of the repair zone, precisely where BrdU labeling was high and where no signals for pepsinogen mRNA or $\mathrm{H}^{+}, \mathrm{K}^{+}$-ATPase protein could be found (Figure 2). The density of CCKBRs increased in the regenerative mucosa with a peak on day 5 (Figure $3 \mathrm{a}$ ). In parallel to wound closure between days 15 and 22, the density and total amount of CCK-BRs decreased substantially, and CCK-BRs were hardly detectable in the mucosal scar on and after day 29. CCK-BRs were selectively localized in the proliferative tissue at the base of the glands, close to the ulcer crater, at days 8 and 15 (Figure 2). The characterization of the CCK-BR in this figure is given by the complete displacement of ${ }^{125} \mathrm{I}-\mathrm{CCK}$ with nanomolar concentrations of unlabeled CCK and gastrin. As internal controls, the adjacent normal mucosa displayed CCK-BRs as well as CCK-ARs, the latter being insensitive to unlabeled gastrin. No CCK-ARs were identified in the repair zone (Figure 2). A highly similar location was obtained when ${ }^{125} \mathrm{I}$-gastrin was used as radio ligand (data not shown).

Typical displacement of bound ${ }^{125} \mathrm{I}-\mathrm{CCK}$ by gastrin, CCK, and YF-476 indicated that we had specifically localized the CCK-BR in the regenerative mucosa (Figure $3 \mathrm{~b}$ ). CCK-ARs and CCK-BRs show nearly identical affinity for sulfated CCK-8, whereas the CCK-BR has an approximately 1,000-fold higher affinity for gastrin than the CCK-AR. The CCK-BR selective antagonist YF-476 displaced the ligand with a high affinity similar to that of CCK-8 or gastrin. In contrast, the CCK-AR selective antagonist L-364,718 displaced the ligand with low affinity only $\left(\mathrm{IC}_{50}>100 \mathrm{nmol} / \mathrm{l}\right)$.

In vitro CCK-BR expression using quantified RT-PCR. To assess the expression of the CCK-BR in the repair zone on the mRNA level, we performed quantified RT-PCR in tissue selectively sampled from the repair zone. Based on histological studies, this regenerative mucosa contained regenerative glands and connective tissue between and below the glands without normal tissue. Selective sampling of regenerative mucosa was impor-

Table 1

Location and time sequence of CCK-AR and CCK-BR during wound healing

\begin{tabular}{|c|c|c|c|c|}
\hline Time point & Histology & Cell proliferation & CCK-BR & CCK-AR \\
\hline & (H\&E staining) & (BrdU staining) & \multicolumn{2}{|c|}{ (Receptor autoradiography) } \\
\hline Intact mucosa $(\text { day } 0)^{A}$ & Intact & Low & Present & Present \\
\hline \multicolumn{5}{|l|}{ Regenerative epithelia } \\
\hline $\begin{array}{l}\text { Days 3-8 } \\
\text { Days 15-22 } \\
\text { Days 22-29 } \\
\text { Days 56-84 }\end{array}$ & $\begin{array}{l}\text { Undiff. glands } \\
\text { Wound closure } \\
\text { Reconstructed glands } \\
\text { Some mature cells }\end{array}$ & $\begin{array}{l}\text { High }^{B} \\
\text { Medium }^{C} \\
\text { Low } \\
\text { Low }\end{array}$ & $\begin{array}{c}\text { High }^{B} \\
\text { Medium } \\
\text { Low } \\
\text { Not detected }\end{array}$ & $\begin{array}{l}\text { Not detected } \\
\text { Not detected } \\
\text { Not detected } \\
\text { Low }\end{array}$ \\
\hline
\end{tabular}

ACell proliferation was detected in the neck region of the intact mucosa. Whereas CCK-BRs were detected in all layers of the intact mucosa, CCK-ARs were

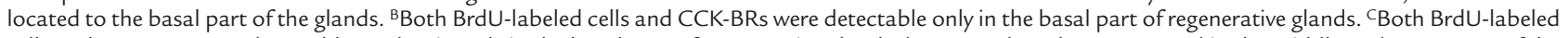
cells and CCK-BRs were detectable predominantly in the basal part of regenerative glands; however, they also reappeared in the middle and upper part of the regenerative glands. H\&E, hematoxylin and eosin. 
tant, since CCK-BR mRNA was expressed more in the normal oxyntic (containing parietal, enterochromaffinlike cells, etc.) than in the regenerative mucosa. Selective sampling of regenerative mucosa was possible only between days 1 and 15, but impossible on days 22, 29, and 84 when the regenerative mucosa was difficult to detect macroscopically. CCK-BR mRNA was not detected by in situ hybridization, but CCK-BR mRNA could be analyzed by quantitative RT-PCR in sampled tissue from the repair zone of the mucosal ulcer margin. CCK-BR mRNA in the regenerative mucosa increased significantly $(P<0.02)$ between days 1 and $15(100 \pm$ $19 \%$, reference value; $156 \pm 21 \% ; 255 \pm 51 \%$; and $434 \pm$ $72 \%$ on days $1,3,8$, and 15 , respectively).

In contrast to the increase of CCK-BR mRNA during healing, the level of the reference gene GAPDH (100 \pm $4 \%, 101 \pm 5 \%, 95 \pm 4 \%$, and $100 \pm 6 \%$ on days $1,3,8$, and 15 , respectively) and pepsinogen (100 $\pm 11 \%, 83 \pm 14 \%$, $75 \pm 15 \%$, and $133 \pm 20 \%$ on days $1,3,8$, and 15 , respectively) remained stable. To compare our results of the expression of CCK-BR mRNA in the repair zone with other well-characterized parameters, the expression of markers of regenerative epithelia (met) and inflammatory cells (COX-2) was quantified. In parallel with the increased number of regenerative mucosa from days $1-15$, we detected significant $(P<0.01)$ increases of mRNA for met in the regenerative mucosa $(100 \pm 12 \%$, reference value; $327 \pm 33 \%$; $362 \pm 36 \%$; and $788 \pm 96 \%$ on days $1,3,8$, and 15$)$. In situ hybridization showed high expression of met in regenerative mucosa and only minimal expression in the normal mucosa. After cryoinjury, the connective tissue between the regenerative epithelia was rapidly infiltrated by inflammatory cells, which are known to express COX-2. Transcripts of COX-2 were not detected by in situ hybridization, but were detected and quantified by RTPCR showing the following significant $(P<0.01)$ changes: $100 \pm 14 \%, 249 \pm 38 \%, 517 \pm 77 \%$, and $93 \pm$ $13 \%$ on days $1,3,8$, and 15 , respectively.

In vivo role of CCK-BRs in the repair zone of the mucosal ulcer margin. In placebo-treated rats, plasma gastrin levels were $50.1 \pm 3 \mathrm{nmol} / 1$. Omeprazole, YF-476, and omeprazole plus YF-476 caused endogenous hypergastrinemia and nearly abolished gastric acid secretion during the observation period. On days 3,8 , and 15 , gastrin plasma levels were $299 \pm 67,332 \pm 41$, and $367 \pm 52 \mathrm{nmol} / 1$ in omeprazole-treated rats, $304 \pm 72$, $345 \pm 61$, and $312 \pm 67 \mathrm{nmol} / 1$ in YF-476-treated rats, and $394 \pm 76,401 \pm 54$, and $389 \pm 48 \mathrm{nmol} / \mathrm{l}$ in omeprazole plus YF-476-treated rats. Injection of gastrin-17 caused exogenous hypergastrinemia. On days 3,8 , and 15 , gastrin plasma levels were $955 \pm 113,967$ \pm 68 , and $775 \pm 137 \mathrm{nmol} / \mathrm{l}$ in gastrin-17, and $1031 \pm$ $132,1113 \pm 201$, and $748 \pm 165 \mathrm{nmol} / \mathrm{l}$ in gastrin-17 plus YF-476-treated rats. Gastrin-17 caused profound gastric hypersecretion during the observation period that was nearly completely abolished by cotreatment with YF-476. Thus, the expected different experimental conditions were achieved.
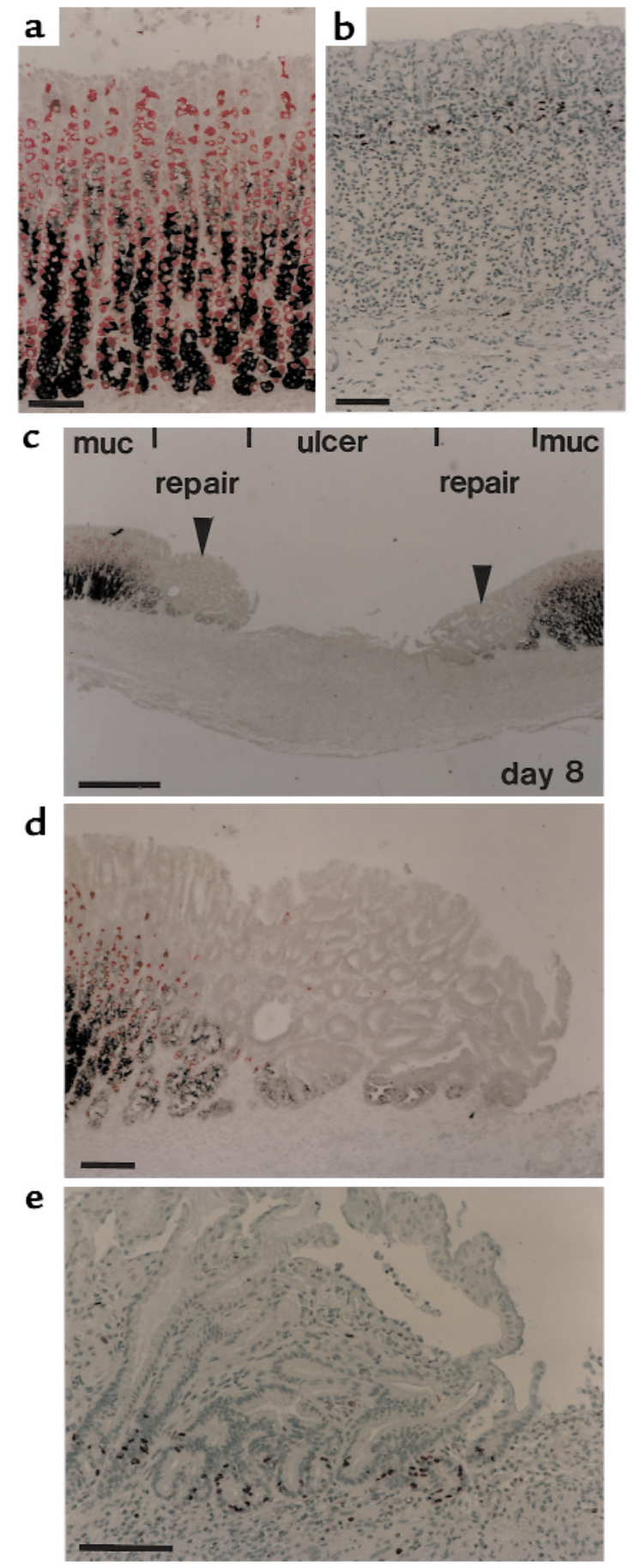

Figure 1

(a and b) Normal oxyntic mucosa from control rats. (c-e) Ulcerated tissue 8 days after ulcer induction showing normal mucosa (muc), regenerative mucosa in the repair zone (arrowheads), and an ulcer crater (ulcer). (a, c, d) In situ hybridization for pepsinogen mRNA (black) and immunostaining for the $\mathrm{H}^{+}, \mathrm{K}^{+}$-ATPase protein (red); (b and e) BrdU immunostaining. In the normal mucosa (a), chief cells (black) are located in the basal mucosa; in contrast, parietal cells (red) are located throughout the mucosa. In the regenerative glands (c and d), no signals for pepsinogen mRNA and $\mathrm{H}^{+}, \mathrm{K}^{+}$-ATPase protein were detected. Whereas BrdU-labeled cells were confined to the neck region in the normal mucosa (b), abundant BrdU labeling was observed in the basal part of regenerative glands close to the ulcer crater (e). Bar, $100 \mu \mathrm{m}$ in a, b, d, e; bar, $500 \mu \mathrm{m}$ in c. 
Figure 2

CCK-BRs in the repair zone. (a-d) Ulcerated tissue, 8 days after ulcer induction. (a) As shown in the hematoxylin-eosin stained section, the ulcer crater is surrounded on both sides by an adjacent repair zone of regenerative mucosa followed by the normal oxyntic mucosa (also see Figure 1c). Bar, $1 \mathrm{~mm}$. (b) Autoradiogram showing total binding of 125I-CCK. It identifies CCK-BRs in the repair zone (arrowheads) and CCK-ARs and CCK-BRs in the normal mucosa on both sides (white stars). (c) Autoradiogram showing ${ }^{125} \mathrm{I}-\mathrm{CCK}$ binding in presence of $10^{-6}$ $M$ gastrin, representing only CCK-ARs. They are absent from the repair zone (arrowheads), but present in the basal half of the glands. (d) Autoradiogram showing nonspecific binding (in the presence of $10^{-6}$ M CCK). (e-h) Repair zone on day 15. (e) As shown in the hematoxylin-eosin stained section, the ulcer crater is re-epithelialized and immature glands have been reconstructed. The repair zone in the center can be distinguished from a transitional zone followed by normal mucosa. (f) Autoradiogram showing total binding of ${ }^{125}$ I-CCK. CCKBRs are found at high density in the repair zone (arrowheads), but only at low density in the transitional zone (white triangles). The mucosa (white stars) expresses CCK-ARs and CCK-BRs. (g) Autoradiogram showing binding of ${ }^{125} \mathrm{I}-\mathrm{CCK}$ in presence of $10^{-6} \mathrm{M}$ gastrin, representing solely CCK-ARs. They are absent from the repair zone (arrowhead), but present in the normal mucosa. (h) Autoradiogram showing nonspecific binding (in presence of $10^{-6} \mathrm{M} \mathrm{CCK}$ ).

In placebo-treated rats, the ulcer diameter, as assessed by repeated video endoscopy, measured $4.64 \pm 0.2 \mathrm{~mm}$ (100\%), $3.77 \pm 0.3 \mathrm{~mm}(81 \%), 2.24 \pm 0.2 \mathrm{~mm}$ (48\%), and $0.93 \pm 0.1 \mathrm{~mm}(20 \%)$ on days $1,3,8$, and 15 , respectively. Quantitative histomorphometric data were nearly identical $(r=0.97)$ to the results of video endoscopy. In the early healing phase, healing was significantly accelerated in all groups containing an acid-inhibitory compound (omeprazole, YF-476, omeprazole plus YF-476, and gastrin-17 plus YF-476) and inhibited by gastrin-17. In the late-healing phase, healing was significantly accelerated in all groups with hypergastrinemia (omeprazole and gastrin-17) and, most importantly, was significantly inhibited in all groups containing YF-476 (Figure 4). Ulcer healing rates (expressed in daily size reduction in percentages) in the late phase (days $8-15$ ) were $4.0 \pm$ $0.2 \%$ in placebo, $4.7 \pm 0.3 \%$ in omeprazole, $3.4 \pm 0.3 \%$ in YF-476, $3.3 \pm 0.3 \%$ in omeprazole plus YF-476, $5.2 \pm$ $0.3 \%$ in gastrin- 17 , and $3.5 \pm 0.2 \%$ in gastrin- 17 plus YF476 treated rats (Figure 4, $\mathrm{c}$ and d). Ulcer sizes on day 15 were dependent on healing rate in the early and late

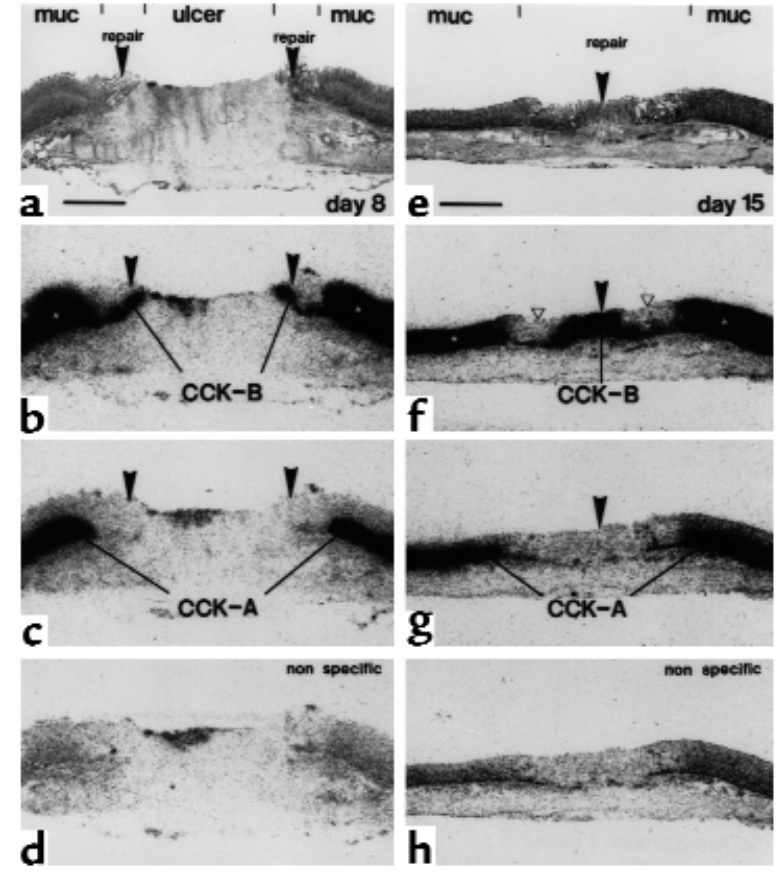

phase and were $20 \pm 2.3 \%$ in placebo-treated, $8 \pm 1.5 \%$ in omeprazole-treated, $12 \pm 1.3 \%$ in YF-476-treated, $11 \pm$ $1.6 \%$ in omeprazole plus YF-476-treated, $16 \pm 1.4 \%$ in gastrin-17-treated, and $10 \pm 2.2 \%$ in gastrin plus YF476 -treated rat groups (Figure 4, a and b).

In the intact oxyntic mucosa, $1.3 \%$ of cells were BrdU labeled (DNA-synthetic phase). Compared with placebo, cell proliferation in the intact oxyntic mucosa on day 15 was slightly $(P<0.05)$ increased by $25 \pm 5 \%$ and $34 \pm 9 \%$ in omeprazole- and gastrin-17-treated rats, respectively. YF-476 completely reversed these effects.

After cryoinjury, cell proliferation in the mucosal ulcer margin was increased severalfold compared with the intact mucosa. Compared with placebo, both omeprazole and gastrin-17 substantially increased cell proliferation in the regenerative mucosal ulcer margin during wound healing. Remarkably, YF-476 also completely reversed both omeprazole- and gastrin17-induced effects on regenerative mucosa (Figure 5).

In placebo-treated animals, the average height of the ulcer margin on days 3,8 , and 15 was $514 \pm 31,506 \pm$ a.

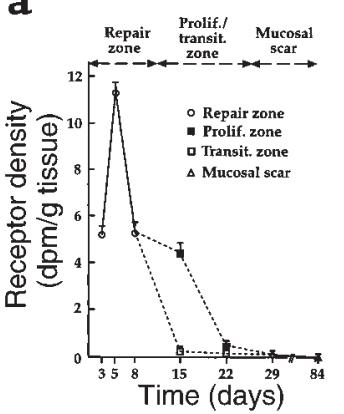

b

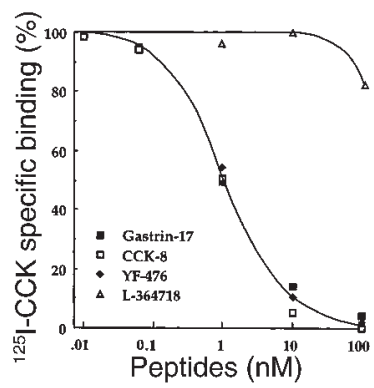

Figure 3

(a) ${ }^{125}$-CCK-specific binding in regenerative mucosa during healing. Whereas labeling was uniform in the repair zone between days 3 and 8 , two zones with different densities for the CCK-BR were detected on days 15 and 22. On days 15 and 22, a proliferative zone in the center of the healing process showed high specific binding, whereas the transitional zone located between the proliferative zone and the normal mucosa showed low specific binding. No or minimal significant binding was detected in the mucosal scar on days 29, 56, and 84 after ulcer induction. Data are expressed as the means \pm SEM. (b) Typical displacement experiment of ${ }^{125}$-CCK for the CCK-BR in the repair zone on day 8 . Tissue sections were incubated with $45 \mathrm{pM}^{125}$-CCK and increasing concentrations of unlabeled, sulfated CCK-8, gastrin, YF-476, and L-364,718. The CCK-BR selective antagonist YF-476 displaces the ligand with a similar high affinity such as CCK-8 or gastrin. In contrast, the CCKAR-selective antagonist L-364,718 displaces the ligand with low affinity only. 
27 , and $489 \pm 20 \mu \mathrm{m}$, and the length of the ulcer margin was $597 \pm 25,818 \pm 29 \mu \mathrm{m}$, and $1349 \pm 31 \mu \mathrm{m}$. In omeprazole-treated rats, the length of the ulcer margin on days 3,8 , and 15 measured $612 \pm 44,932 \pm 47$, and $1594 \pm 66 \mu \mathrm{m}$, with significant $(P<0.02$ vs. placebo $)$ differences on days 8 and 15 .

The thickness of the intact oxyntic mucosa on day 15 was $612 \pm 13 \mu \mathrm{m}$ in placebo-treated rats and was significantly $(P<0.05)$ increased in omeprazole- and gastrin-17-treated rats by $19 \%$ and $24 \%$, respectively. In contrast, the thickness of the mucosa was significantly $(P<0.05)$ decreased in YF-476-treated, omeprazole plus YF-476-treated, and gastrin-17 plus YF-476-treated rats by $17 \%, 14 \%$, and $12 \%$, respectively.

There were no postoperative complications in the entire study period, and the rats showed an average weight gain of approximately 2 g per day, with no differences between the treatment groups.

\section{Discussion}

This study provides morphological and functional evidence for the significance of gastrin and the CCK-BR in gastric wound healing. Morphologically, CCK-BRs were shown to be expressed and localized to the regenerative mucosa in the repair zone adjacent to the ulcer crater. This high expression was limited in time, and the time sequence of expression and location of CCKBRs was closely related to the proliferative activity of the regenerative mucosa. In vivo, rats with endogenous (induced by omeprazole) and exogenous (induced by injected gastrin-17) hypergastrinemia showed significant trophic effects on the regenerative mucosa of the ulcer margin. The highly potent CCK-BR antagonist YF-476, however, completely abolished these effects. Therefore, these data suggest that CCK-BRs in the regenerative mucosa enhance trophic effects during gastric wound healing.

The density of CCK-BRs paralleled the number of repair cells in the DNA-synthetic phase (BrdU-labeled cells), which between days 3 and 15 were highest at the lower part of the glands, close to the ulcer crater. The number of BrdU-labeled cells and the density of CCKBRs decreased markedly after the reepithelialization of the ulcer crater between days 15 and 22. This rapid decrease of the expression of CCK-BRs in the repair zone after complete reepithelialization of the ulcer crater suggests that the upregulation of the CCK-BR may be caused by mediators present in the ulcerated region.

Our receptor-autoradiographic data clearly localized CCK-BRs to the regenerative mucosa. Since relevant expression of CCK-BRs on fibroblasts, endothelial cells, or inflammatory cells has not been reported, the most probable location for our CCK-BR binding sites in this model are the epithelial cells. This conclusion is based on circumstantial evidence since we cannot locate the CCKBRs to a specific cell type using our receptor-autoradiographic technique. The lack of differentiated epithelial cells in the repair zone, combined with a rapid increase of both the density of CCK-BRs and the number of
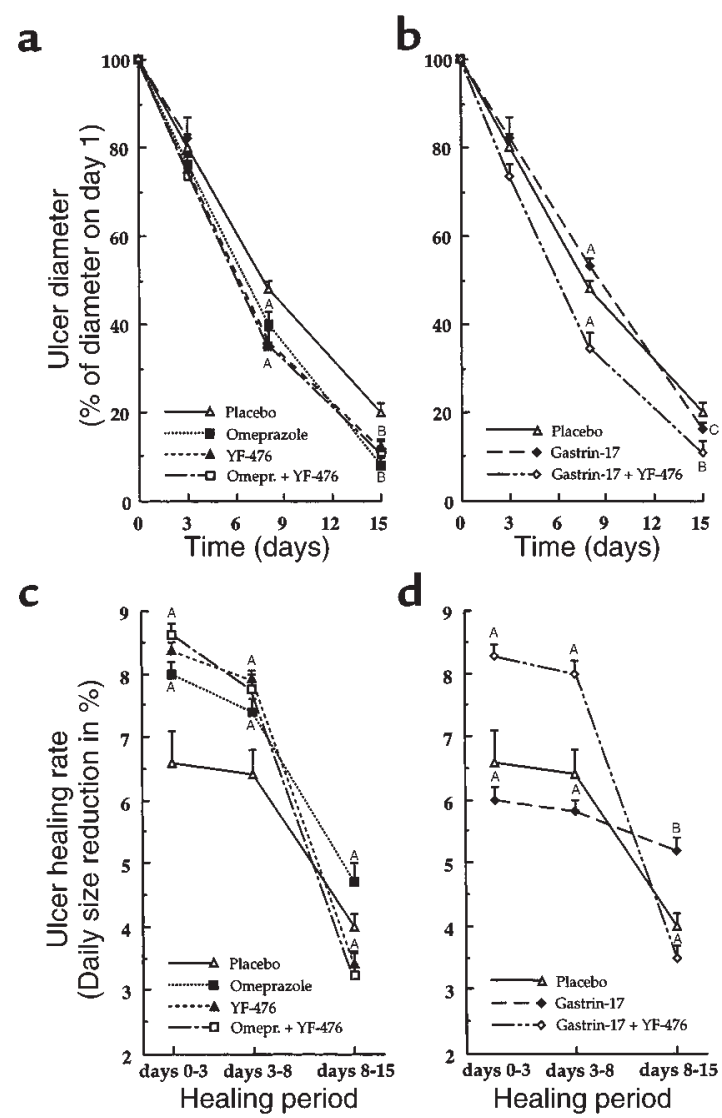

\section{Figure 4}

Ulcer diameter time curve ( $\mathbf{a}$ and $\mathbf{b}$ ) and ulcer-healing rate time curve (c and d). Compared with placebo (open triangles), omeprazole (filled squares) accelerated healing both in the early (days $0-8$ ) and late (days 8-15) phase (a and c). YF-476 (filled triangles) also accelerated healing in the early phase, but significantly delayed healing in the late phase (a and $\mathbf{c}$ ). Gastrin-17 (filled diamonds) delayed healing in the early phase, but profoundly accelerated healing in the late phase (b and d). Healing curves of YF-476, omeprazole plus YF-476 (open squares), and gastrin-17 plus YF-476 (open diamonds) were nearly identical (see text for exact data). Data are expressed as the means $\pm \mathrm{SEM}$. ${ }^{\mathrm{A} P}<0.05,{ }^{\mathrm{B}} P<0.01$, and ${ }^{\mathrm{C}} P=0.33$ vs. placebo.

undifferentiated epithelial cells in the ulcer margin, suggest that CCK-BRs are located on regenerative epithelia that derive from progenitor cells (34). In the normal mucosa, CCK-BRs have been located to endocrine (35) and progenitor cells found in the neck region of the glands by various methods, including those on CCK$\mathrm{BR}$-deficient mice generated by gene targeting $(36,37)$. The number of CCK-BRs on progenitor cells of the normal gland is below the detection level of the present morphological techniques. Progenitor cells from the neck region, however, proliferate rapidly after injury to maintain integrity of the mucosa (34). Since the regenerative cells derived from progenitor cells expand, CCK-BRs may become detectable at that time and can be characterized on proliferating mucosa by receptor autoradiography. This concept is also supported by our in vivo studies showing that cell proliferation in the mucosal ulcer margin and in the intact mucosa can be stimulated by 

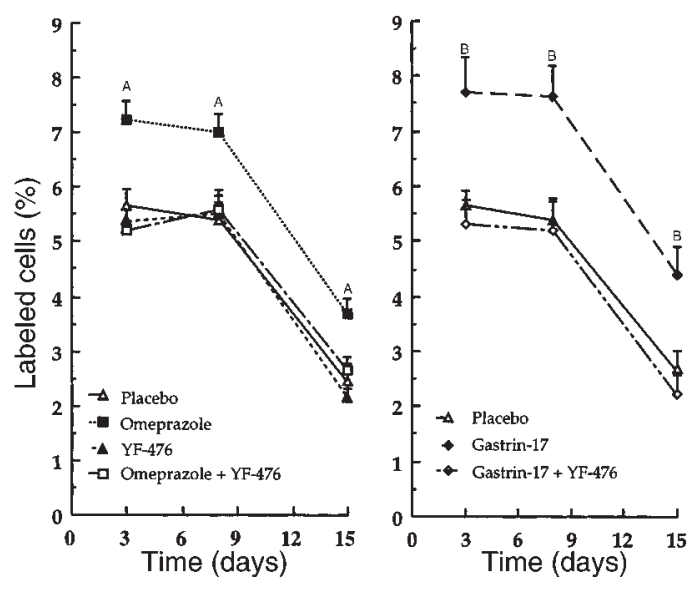

Figure 5

BrdU-labeled cells in the mucosal ulcer margin (repair zone) adjacent to the ulcer crater 3,8 , and 15 days after ulcer induction. Compared with placebo (open triangles), omeprazole (filled squares) and gastrin-17 (filled diamonds) significantly increased cell proliferation in the mucosal ulcer margin. In contrast, YF-476 (filled triangles) had no significant effect on cell proliferation, and coadministration of YF-476 to omeprazole (open squares) or gastrin-17 (open diamonds) completely reversed the proliferative effects of drug-induced hypergastrinemia. Data are expressed as the means \pm SEM. ${ }^{A} P<0.02,{ }^{B} P<0.002$ vs. placebo.

endogenous and exogenous hypergastrinemia and inhibited by the CCK-BR antagonist YF-476.

Since CCK-BRs are located in the normal oxyntic mucosa on several differentiated cell types, including parietal and enterochromaffin-like cells, it is important to exclude the possibility that these differentiated cells are present in the repair zone adjacent to the ulcer crater. After cryoinjury, chief cells showed a rapid and profound decrease in the expression of pepsinogen mRNA and CCK-AR protein in the repair zone. In parallel, parietal cells that normally express both $\mathrm{H}^{+}, \mathrm{K}^{+}-$ ATPase and several G-protein-coupled receptors, including the somatostatin receptor and the CCK-BR, also showed a rapid decrease in the expression of these proteins (26). Endocrine cells including enterochromaffin-like cells, which normally express CCK-BRs, also could not be detected within the repair zone (6).

CCK-BR mRNA was not detected using in situ hybridization, but the expression of CCK-BRs was quantified by RT-PCR in tissue carefully sampled from the repair zone. These data support our receptor autoradiographic data, which localized CCK-BR to the regenerative mucosa. Interestingly, the time sequence of expression of the hepatocyte growth-factor receptor met seems to be similar to that of the CCK-BR (4). Transcripts for met that can be localized by in situ hybridization are weakly expressed in the neck region of the normal mucosa; however, they are strongly expressed in the lower part of regenerative glands (4), with a substantial increase in expression between days 1 and 15 .

Further confirmation for the regulatory roles of gastrin has been acquired from our in vivo studies. In the regenerative mucosa, a marked mitogenic effect of omeprazole and gastrin-17 was detected. In the present in vivo study, cell proliferation in the mucosal ulcer margin was increased by omeprazole and gastrin-17. YF476, however, completely inhibited these effects. The effect of YF-476 is most likely due to the inhibition of CCK-BR-mediated trophic actions of gastrin that are best detected in hypergastrinemic rats. These data clearly indicate that other posttranslational intermediates of gastrin, such as glycine-extended gastrin and other receptors, are unlikely to be relevant to this mitogenic effect. This further supports our hypothesis that CCKBRs are localized on regenerative epithelia and that these receptors are functionally intact and relevant.

In our ulcer-healing model, healing in the early phase (days $0-8$ ) is dependent predominantly on cell migration over the ulcer crater, which is stimulated by all acid-inhibitory drugs (i.e., omeprazole and YF-476) and, conversely, inhibited by all acid-stimulating compounds (i.e., gastrin-17) (3). Ulcer healing in the late phase is, however, dependent predominantly on epithelial cell proliferation in the mucosal ulcer margin, and gastric acidity has no relevant effects (3). Thus, in the present study, ulcer healing in the early phase was accelerated by omeprazole and YF-476 and was inhibited by gastrin-17. Hypergastrinemia (caused by omeprazole and gastrin-17) substantially increased epithelial cell proliferation in the mucosal ulcer margin and accelerated gastric ulcer healing in the late phase; in contrast, YF-476 delayed gastric ulcer healing in the late phase. Most importantly, YF-476 completely reversed omeprazole and gastrin-17-mediated effects on epithelial cell proliferation and ulcer healing rate.

Gastric acid has a major impact on the gastric wound healing process, directly by affecting cell migration over the ulcer crater and indirectly by changing the effects of various acid-unstable growth factors $(3,4)$. Since it is impossible to dissociate in vivo the CCK-BR-mediated effects on epithelial cell proliferation and gastric acid secretion, it is difficult to assess the exact impact of the CCK-BR on gastric wound healing under physiological conditions (i.e., physiological gastric acid secretion and gastrin plasma levels).

As a control, we also assessed the drug effects on plasma gastrin levels and on cell proliferation in the normal oxyntic mucosa $(10,11)$. Omeprazole and YF-476 caused approximately a sevenfold increase in plasma gastrin levels and a slight, but significant, increase in cell proliferation of the oxyntic mucosa. Gastrin-17 caused approximately a 20 -fold increase in plasma gastrin levels and also a significant increase in cell proliferation of the oxyntic mucosa. YF-476 abolished the trophic effects of endogenous and exogenous hypergastrinemia and caused atrophy of the intact oxyntic mucosa.

The growth-promoting effect of gastrin (18-20) has generated substantial interest because of potential side effects such as gastric or colorectal tumor growth during hypergastrinemia induced by long-term treatment with gastric acid-inhibitory drugs $(38,39)$. The intra- 
cellular signaling pathways of the CCK-BR have been roughly characterized in enterochromaffin-like cells in which CCK-BRs mediate their proliferative action by activating the Ras-MAP kinase pathway (40).

The complete healing of wounds is the final step in a highly regulated response to injury. Although many of the molecular mediators and cellular events of healing are known, their manipulation for the enhancement and acceleration of wound closure has not yet been proven to be practical. Earlier studies demonstrated that a variety of peptide growth factors promote cell proliferation in the mucosa, angiogenesis, and ulcer healing (2-4), but no small nonpeptide molecule, which can be systemically applied, has yet been shown to enhance repair mechanisms. Stimulation of the CCK-BR during wound healing by high gastrin levels caused by treatment with proton pump inhibitors is therefore an attractive strategy for improvement of wound healing in the upper gastrointestinal tract.

In conclusion, the location and time sequence of expression of the CCK-BR in regenerating mucosal tissue during wound healing in the oxyntic mucosa suggest a significant role for the CCK-BR in the healing process. The increase in cell proliferation in hypergastrinemic rats, which can be profoundly inhibited by the CCK-BR antagonist YF-476, supports the concept that gastrin can enhance trophic actions on the regenerative mucosa through the CCK-BR.

\section{Acknowledgments}

This work was supported by a grant from the Swiss National Science Foundation (32-51099.97).

1. Martin, P. 1997. Wound healing: aiming for perfect skin regeneration. Science. 276:75-81.

2. Wright, N.A., Pike, C., and Elia, G. 1990. Induction of a novel epidermal growth factor-secreting cell lineage by mucosal ulceration in human gastrointestinal stem cells. Nature. 343:82-85.

3. Schmassmann, A., et al. 1995. Influence of acid and angiogenesis on kinetics of gastric ulcer healing in rats: interaction with indomethacin. Am. J. Physiol. 268:G276-G285.

4. Schmassmann, A., et al. 1997. Roles of hepatocyte growth factor and its receptor met during gastric ulcer healing in rats. Gastroenterology. 113:1858-1872.

5. Bamberg, K., et al. 1994. In situ hybridization of mRNA for the gastric $\mathrm{H}^{+}, \mathrm{K}^{+}$-ATPase in the rat oxyntic mucosa. Biochim. Biophys. Acta. 1190:355-359.

6. Helander, H.F., Weijdegard, B., and Bamberg, K. 1996. The expression of pepsinogen $\mathrm{C}$ mRNA in normal gastroduodenal mucosa and the gastric ulcer margin of the rat. Histochem. Cell Biol. 105:163-169.

7. Lamberts, S.W., Krenning, E.P., and Reubi, J.C. 1991. The role of somatostatin and its analogs in the diagnosis and treatment of tumors. Endocr. Rev. 12:450-482.

8. Ashcroft, G.S., et al. 1997. Estrogen accelerates cutaneous wound healing associated with an increase in TGF- $\beta 1$ levels. Nat. Med. 3:1209-1215.

9. Montesinos, M.C., et al. 1997. Wound healing is accelerated by agonists of adenosine $\mathrm{A}_{2}\left(\mathrm{G}_{\alpha \mathrm{s}}\right.$-linked) receptors. J. Exp. Med. 186:1615-1620.

10. Nishida, A., et al. 1995. Gastrin receptor antagonist YM022 prevents hypersecretion after long-term acid suppression. Am. J. Physiol. 269:G699-G705

11. Ding, X.Q., Lindström, E., and Hakanson, R. 1997. Evaluation of three novel cholecystokinin-B/gastrin receptor antagonists: a study of their effects on rat stomach enterochromaffin-like cell activity. Pharmacol. Toxicol. 81:232-237.

12. Amagase, K., Ikeda, K., and Okabe, S. 1999. Antisecretory and ulcer healing effects of S-0509, a novel CCK-B/gastrin receptor antagonist, in rats. Dig. Dis. Sci. 44:879-888.

13. Walan, A., et al. 1989. Effect of omeprazole and ranitidine on ulcer heal- ing and relapse rates in patients with benign gastric ulcer. N. Engl. J. Med. 320:69-75

14. Yeomans, N.D., et al. 1998. A comparison of omeprazole with ranitidine for ulcers associated with nonsteroidal antiinflammatory drugs. N. Engl. J. Med. 338:719-726.

15. Wank, S.A., Pisegna, J.R., and de Weerth, A. 1992. Brain and gastrointestinal cholecystokinin receptor family: structure and functional expression. Proc. Natl. Acad. Sci. USA. 89:8691-8695.

16. Kopin, A.S., et al. 1992. Expression cloning and characterization of the canine parietal cell gastrin receptor. Proc. Natl. Acad. Sci. USA. 89:3605-3609.

17. Reubi, J.C., et al. 1997. Localization of cholecystokinin A and cholecystokinin B/gastrin receptors in the human stomach and gallbladder. Gastroenterology. 112:1197-1205.

18. Wank, S.A. 1998. G protein-coupled receptors in gastrointestinal physiology. I. CCK receptors: an exemplary family. Am. J. Physiol. 274:G607-G613.

19. Li, H., and Helander, H.F. 1996. Hypergastrinemia increases proliferation of gastroduodenal epithelium during gastric ulcer healing in rats. Dig. Dis. Sci. 41:40-48.

20. Takeuchi, K., and Johnson, L.R. 1986. Effect of cell proliferation on healing of gastric and duodenal ulcers in rats. Digestion. 33:92-100.

21. Baldwin, G.S. 1994. Antiproliferative gastrin/cholecystokinin receptor antagonists target the $78-\mathrm{kDa}$ gastrin binding protein. Proc. Natl. Acad. Sci. USA. 91:7593-7597.

22. Bold, R.J., Ishizuka, J., Townsend, C.M., Jr., and Thompson, J.C. 1994. Gastrin stimulates growth of human cancer cells via a receptor other than CCK-A or CCK-B. Biochem. Biophys. Res. Commun. 202:1222-1226.

23. Singh, P., Owlia, A., Espeijo, R., and Dai, B. 1995. Novel gastrin receptors mediate mitogenic effects of gastrin and processing intermediates of gastrin on Swiss 3T3 fibroblasts. Absence of detectable cholecystokinin (CCK)-A and CCK-B receptors. J. Biol. Chem. 270:8429-8438.

24. Seva, C., Dickinson, C.J., and Yamada, T. 1994. Growth-promoting effects of glycine-extended progastrin. Science. 265:410-412.

25. Tsutsui, S., et al. 1997. Induction of heparin binding epidermal growth factor-like growth factor and amphiregulin mRNAs by gastrin in the rat stomach. Biochem. Biophys. Res. Commun. 235:520-523.

26. Reubi, J.C., Waser, B., Schmassmann, A., and Halter, F. 1994. Persistent lack of somatostatin receptors in gastric mucosa of healing ulcers in rats. Gastroenterology. 107:339-346.

27. Mizuno, H., et al. 1997. Induction of cyclooxygenase 2 in gastric mucosal lesions and its inhibition by the specific antagonist delays healing in mice. Gastroenterology. 112:387-397.

28. Schmassmann, A., et al. 1998. Effects of inhibition of prostaglandin endoperoxide synthase- 2 in chronic gastro-intestinal ulcer models in rats. Br. J. Pharmacol. 123:795-804.

29. Breitschopf, H., Suchanek, G., Gould, R.M., Colman, D.R., and Lassmann, H. 1993. In situ hybridisation with digoxigenin-labeled probes: sensitive and reliable detection method applied to myelinating rat brain. Acta Neuropathol. 84:581-587.

30. Ichihara, Y., Sogawa, K., Morohashi, K., Fujii-Kuriyama, Y., and Takahashi, K. 1986. Nucleotide sequence of a nearly full-length cDNA coding for pepsinogen of rat gastric mucosa. Eur. J. Biochem. 161:7-12.

31. Reubi, J.C., et al. 1990. Detection of somatostatin receptors in surgical and percutaneous needle biopsy samples of carcinoids and islet cell carcinomas. Cancer Res. 50:5969-5977.

32. Reubi, J.C., Schaer, J.C., and Waser, B. 1997. Cholecystokinin (CCK)-A and CCK-B/gastrin receptors in human tumors. Cancer Res. 57:1377-1386.

33. Tatsuta, M., et al. 1988. Effect of cimetidine on inhibition by tetragastrin of carcinogenesis induced by $N$-Methyl- $N^{\prime}$-nitro- $N$-nitrosoguanidine in Wistar rats. Cancer Res. 48:1591-1595.

34. Karam, S.M., and Leblond, C.P. 1993. Dynamics of epithelial cells in the corpus of the mouse stomach. I. Identification of proliferative cell types and pinpointing of the stem cell. Anat. Rec. 236:259-279.

35. Helander, H.F., Wong, H., Poorkhalkali, N., and Walsh, J.H. 1997. Immunohistochemical localization of gastrin/CCK-B receptors in the dog and guinea-pig stomach. Acta Physiol. Scand. 159:313-320.

36. Nagata, A., et al. 1996. G protein-coupled cholecystokinin-B/gastrin receptors are responsible for physiological cell growth of the stomach mucosa in vivo. Proc. Natl. Acad. Sci. USA. 93:11825-11830.

37. Langhans, N., et al. 1997. Abnormal gastric histology and decreased acid production in cholecystokinin-B/gastrin receptor-deficient mice. Gastroenterology. 112:280-286.

38. Rehfeld, J.F. 1995. Gastrin and colorectal cancer: a never-ending dispute? Gastroenterology. 108:1307-1310.

39. Thorburn, C.M., et al. 1998. Gastrin and colorectal cancer: a prospective study. Gastroenterology. 115:275-280.

40. Kinoshita, Y., et al. 1998. Comparison of the signal transduction pathways activated by gastrin in enterochromaffin-like and parietal cells. Gastroenterology. 115:93-100 